\section{Four rooted maxillary third molar: a case report of a rare clinical presentation}

\author{
Bamgbose B. Olamide, ${ }^{1 *}$ Mohammad A. Kaura, ${ }^{2}$ Anas I. Yahaya ${ }^{3}$
}

\title{
Abstract
}

Objective: Maxillary molars are known to have three roots (two buccal and one palatal) and the occurrence of four-rooted maxillary molars is uncommon. Maxillary teeth with accessory roots have been classified into four categories based on root configurations. The presence of an accessory root may complicate an otherwise routine exodontia of the maxillary third molar, especially because of the proximity of the floor of the maxillary antrum and the maxillary tuberosity.

Methods: A 35 years old male patient was referred to the Oral Diagnostic Sciences Clinic from the Ear Nose and Throat (ENT) Clinic on account of right-sided facial pain of one-month duration. Intraoral examination, revealed a carious maxillary right third molar that was tender to percussion. Periapical radiograph revealed large coronal radiolucency involving the distal half of the tooth with extension into the distal pulp horn. The portrayed outlines of the roots showed two buccal roots and the outlines of what appears to be two straight palatal roots.

Results: Socket extraction of the maxillary third molar under local anaesthesia.

Conclusion: In clinical practice of endodontics and oral surgery, it is good practice to anticipate the likely presence of an accessory root on a maxillary molar in order to avoid post-operative complications following treatment.
'Department of Oral Diagnostic Sciences, Faculty of Dentistry, Bayero University Kano/Aminu Kano Teaching Hospital Kano, Kano, Nigeria

${ }^{2}$ Department of Oral and Maxillofacial Surgery, Bayero University Kano/Aminu Kano Teaching Hospital Kano, Kano, Nigeria

${ }^{3}$ Department of Anatomy, Faculty of Basic Medical Sciences, Bayero University Kano, Kano, Nigeria

\section{*Corresponding to:}

Bamgbose B. Olamide, Department of Oral Diagnostic Sciences, Faculty of Dentistry, Bayero University Kano/ Aminu Kano Teaching Hospital Kano, Kano, Nigeria

drtundebamgbose@yahoo.com

Received: 1 June 2018

Revised: 2 June 2018

Accepted: 23 July 2018

Available online 1 August 2018

Keywords: Four-rooted maxillary third molar, Periapical radiograph, Exodontia

Cite this Article: Olamide BB, Kaura MA, Yahaya Al. 2018. Four rooted maxillary third molar: a case report of a rare clinical presentation. Journal of Dentomaxillofacial Science 3(2): 126-128. D0I: 10.15562/jdmfs.v3i2.751

\section{Introduction}

Maxillary molars are known to have three roots. two buccal and one palatal. The occurrence of fourrooted maxillary molars is uncommon and the incidence is reported to be as low as $0.4 \%$ for the second molars, $0.9 \%$ for the maxillary first molars and $1.8 \%$ for the maxillary third molars. ${ }^{1-4}$ Extra root formation may occur either by division or folding of the Hertwig epithelial root sheath. This may lead to the formation of two similar roots or an independent root. ${ }^{5}$

Maxillary teeth with accessory roots have been classified by Christie et al. ${ }^{6}$ into four categories based on root configurations. The type I maxillary molars have two widely divergent palatal roots and four separate apices are seen on the radiograph. The type II maxillary molars have four separate, short roots with blunt apices. The type III maxillary molars have constricted root morphology with the mesiobuccal, mesiopalatal and distopalatal roots coalesced in a blub of root dentin, while the distobuccal root stands alone. The type IV maxillary molars have four roots but the accessory root is fused with the mesiobuccal root up to the apical level.

Carlsen et al. ${ }^{7}$ further classified maxillary molars based on the relationship of the accessory root to the crown. They classified the four-rooted molars as radix mesiolingualis and radix distolingualis. The radix mesiolingualis is directly related to the mesiopalatal part of the maxillary molar while radix distolingualis has an accessory root with affinity for the distopalatal portion of the maxillary molar. ${ }^{7}$

The presence of an accessory root may complicate an otherwise routine exodontia of the maxillary third molar, especially because of the proximity of the floor of the maxillary antrum and the maxillary tuberosity.

We report a case of four-rooted maxillary third molar that presented at our facilities for diagnosis and routine socket extraction.

\section{Case Report}

A 35 years old male patient was referred to the Oral Diagnostic Sciences Clinic from the Ear Nose and Throat (ENT) Clinic, upon ruling out any infection involving the ear, nose, or throat, for review and management on account of right-sided facial pain of one month duration. The pain was severe, intermittent and disturbed daily activities. The pain involved the right side of the face, ear, and head.

Upon intraoral examination, the mouth opening was adequate and oral hygiene was good. The patient had generalized fluorosis and a full complement of teeth. A carious maxillary right third molar was observed. The tooth was tender to percussion. 
There was no associated swelling or purulent discharge. All the intraoral soft tissues appeared clinically healthy.

Periapical radiograph revealed large coronal radiolucency involving the distal half of the tooth with extension into the distal pulp horn. The appearance was suggestive of a large carious lesion with pulpal involvement. The portrayed outlines of the roots showed two buccal roots and the outlines of what appears to be two straight palatal roots with uniform thickness of the periodontal ligament space. The coronoid process is clearly demonstrated on the periapical radiograph figure 1 . The patient was scheduled for routine extraction of the maxillary right third molar under local anaesthesia.

Posterior superior alveolar and greater palatine nerve blocks were administered using 2\%

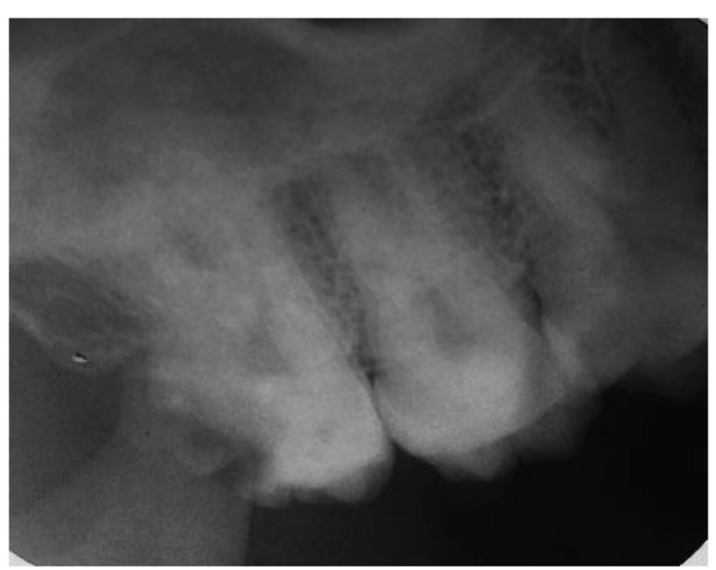

Figure 1 Periapical radiograph reveals large coronal radiolucency involving the distal half of the tooth with extension into the distal pulp horn. The portrayed outlines of the roots demonstrate two buccal roots and two straight palatal roots with uniform thickness of the periodontal ligament space
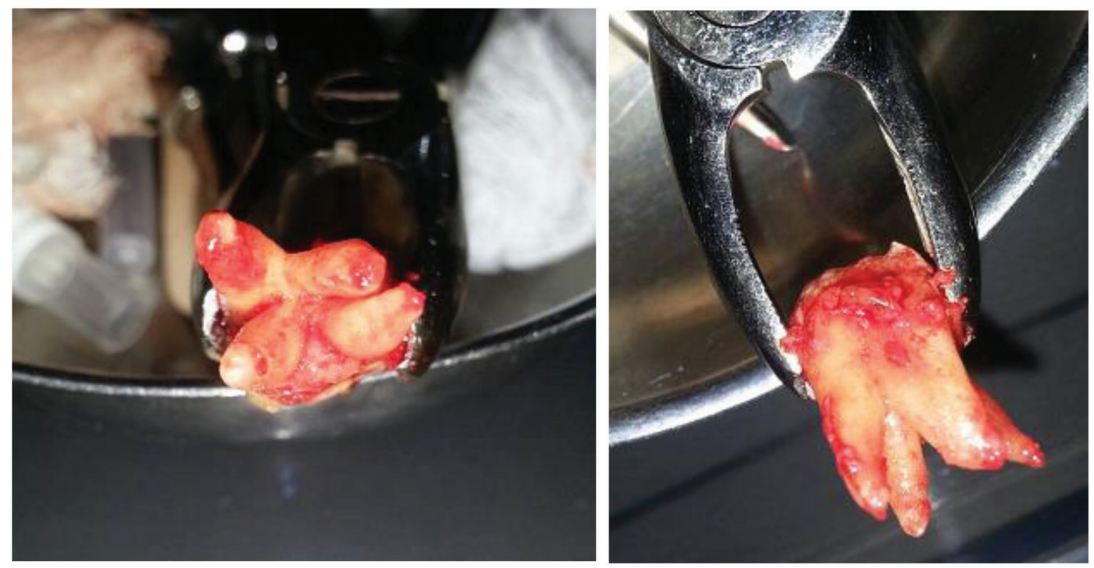

Figure 2 The extracted maxillary right third molar shows four distinct and fully formed roots (two buccal and two palatal) lignocaine with 1:80.000 epinephrine. Transalveolar extraction was attempted but it proved difficult. The mucoperiosteal flap was raised and the height of the buccal cortical plate was reduced using a slow handpiece and round bur under constant $0.9 \%$ normal saline irrigation. This was done to prevent fracture of the maxillary tuberosity or inadvertent exposure of the maxillary antrum that may eventually lead to oroantral fistula complication. The extraction of the maxillary right third molar was uneventful and there were no postoperative complications. Clinical observation revealed a four-rooted maxillary third molar and it was our understanding that the root configuration was responsible for the difficulty during the procedure figure 2. Post-operative analgesics were prescribed and the one-week postoperative review was uneventful.

\section{Discussion}

The incidence of four-rooted maxillary molars has been reported to be as low as $0.4-1.8 \%{ }^{1-3}$ Accessory roots are, therefore, not routinely anticipated on maxillary molars when planning either endodontic or oral surgical procedures. Inadequate preoperative planning and poor radiologic review may result in unanticipated complications when accessory roots are present. The observation of coronala and radicular landmarks is important for the detection of accessory roots. Maxillary molars with accessory roots usually present with a prominent or an accessory cusp with an associated relatively large crown size. ${ }^{7,8}$ Periodontal probing of the cervical third of the palatal root may also reveal radicular grooves or root bifurcation which may suggest an accessory palatal root. ${ }^{9}$ It is not always easy to detect accessory roots in the posterior maxillary region on periapical radiographs because of the superimposition of anatomic structures such as zygomatic process of the maxilla, floor of the antrum and the coronoid process of the mandible. In addition, the periapical radiograph is a two dimensional representation of a three dimensional structure. In the present case, the periapical radiograph revealed the outline of two prominent buccal roots and two palatal roots with distinct periodontal ligament spaces. Anatomical structures, such as zygomatic process of the maxilla, floor of the maxillary sinus, and the coronoid process of the mandible were also observed but these structures did not obscure the visualization of the root outlines. The palatal 
The presence of two periodontal ligament spaces around a single root, periodontal ligament space crossing over a root and inability to identify a palatal root between the mesiobuccal and distobuccal roots are other radiologic features that may suggest the presence of an accessory root on a maxillary molar. $^{8}$

In a practice where facilities are available, Cone Beam Computed Tomography (CBCT) will be indicated to further investigate a maxillary molar suspected to have an accessory root. The CBCT is able to localize the accessory root and provide a three-dimensional rendition of the root canal and root morphology for endodontic and oral surgical purposes.

The present case represents a type II maxillary molar with four separate roots that are short and run parallel to each other, according to the classification presented by Christie et al. ${ }^{6}$

In clinical practice of endodontics and oral surgery, it is good practice to anticipate the likely presence of an accessory root on a maxillary molar in order to avoid post operative complications following treatment.

\section{Conclusion}

In clinical practice of endodontics and oral surgery, it is good practice to anticipate the likely presence of an accessory root on a maxillary molar in order to avoid post operative complications following treatment.

\section{Acknowledgment}

The author would like to thank the patient who has been willing to share his case for reported and for his cooperation to come for control treatment.

\section{Conflict of Interest}

The authors expressed no conflicts of interest with this study.

\section{References}

1. Libfeld H, Rostein I. Incidence of four-rooted maxillary second molars: literature review and radiographic survey of 1200 teeth. J Endod 1989;15: 129-131.

2. Kottoor J, Velmurugan N, Ballal S, Roy A. Four-Rooted maxillary first molar having c-shaped palatal root canal morphology evaluated using cone-beam computerized tomography: a case report. Oral Surg Oral Med Oral Pathol Oral Radiol Endod 2011;111: e41-45.

3. Vertucci FJ. Root canal morphology and its relationship to endodontic procedures. Endod Topics 2005;10: 3-29.

4. Cleghorn BM, Christie WH, Dong CC. Root and root canal morphology of the human permanent maxillary first molar: a literature review. J Endod 2006;32: 813-821

5. Turp JC, Alt KW. Anatomy and morphology of human teeth. In: Alt KW, Rosing FW, Teschler-Nicola M, editors. Dental Anthropology. Fundamentals, Limits and prospects. Austria: Springer; 1998. p. 71-94.

6. Christie WH, Peikoff MD, Fogel HM. Maxillary molars with two palatal roots: a retrospective clinical study. J Endod 1991;17: 80-84

7. Carlsen O, Alexendersen V. Radix mesiolingualis and radix distolingualis in a collection of permanent maxillary molars. Acto Odontol Scand 2000;58: 229-236

8. Ahmed HM, Abbott PV. Accessory roots in maxillary molar teeth: a review and endodontic considerations. Aust Dent J 2012;57: 123-131

9. Calberson FL, De Moor RJ, Deroose CA. The radix entomolaris and paramolaris: clinical approach in endodontics. J Endod 2007;33: 58-63.

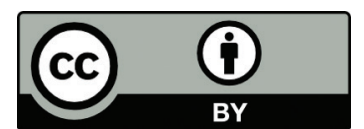

This work is licensed under a Creative Commons Attribution 